\title{
SHAPE-PRESERVING SOLUTIONS OF THE TIME-DEPENDENT DIFFUSION EQUATION*
}

\author{
BY \\ FRANK S. HAM \\ General Electric Research Laboratory \\ Schenectady, New York
}

\begin{abstract}
Exact solutions to the time-dependent diffusion equation are exhibited which correspond to the diffusion-limited growth of ellipsoidal precipitate particles with constant shape and dimensions proportional to the square root of the time. The asymmetry of the diffusion field in these solutions is consistent with the preservation of the particle's shape during growth even if the diffusivity is anisotropic. Limiting cases for simpler geometries are derived and shown to be in agreement with previously known results for radially symmetric particles and isotropic diffusion. Similar solutions for hyperboloidal surfaces are exhibited and generalizations are considered analogous to those discussed by Danckwerts for one-dimensional diffusion.

I. Introduction. Exact solutions of the time-dependent diffusion equation are known [1-5] which correspond to the growth of radially symmetric particles in one, two, and three dimensions with radius proportional to the square root of the time. The initial density of diffusing material is uniform and the density at the surface of the particle is specified. The rate of growth is directly proportional to the concentration gradient at the surface. It is the purpose of this paper to show that these solutions are special cases of a more general class of shape-preserving solutions appropriate to ellipsoidal and hyperboloidal surfaces. The generalized boundary condition for the rate of growth is that the velocity of motion of the surface along its normal is proportional to the normal component of the concentration gradient. These more general solutions are of interest in connection with studies of diffusion-limited phase transformations for which the interface between the phases is not radially symmetric or for which the diffusivity is anisotropic, or both.
\end{abstract}

II. General solutions. We shall examine only those solutions of the isotropic timedependent diffusion equation

$$
\frac{\partial \rho}{\partial t}=D \nabla^{2} \rho
$$

which are functions of the reduced variables

$$
\begin{aligned}
u & =x t^{-1 / 2}, \\
v & =y t^{-1 / 2}, \\
w & =z t^{-1 / 2},
\end{aligned}
$$

where $x, y, z$ are Cartesian coordinates of position and $t$ is the time. In (1) $\rho$ is the density of diffusing material and $D$ an appropriate diffusivity, assumed for the present to be a scalar. Introducing the vector $\mathrm{s}=(u, v, w)$ and the gradient operator $\nabla_{\mathbf{C}}=(\partial / \partial u$

*Received April 2, 1958; revised manuscript received July 3, 1958. 
$\partial / \partial v, \partial / \partial w)$, we find that (1) becomes

$$
-\frac{1}{2} \mathrm{~s} \cdot \nabla_{s} \rho=D \nabla_{s}^{2} \rho .
$$

We now introduce general ellipsoidal coordinates according to the relations [6]

$$
\begin{aligned}
u & =\left[\left(\xi_{1}^{2}-a^{2}\right)\left(\xi_{2}^{2}-a^{2}\right)\left(\xi_{3}^{2}-a^{2}\right) / a^{2}\left(a^{2}-b^{2}\right)\right]^{1 / 2} \\
v & =\left[\left(\xi_{1}^{2}-b^{2}\right)\left(\xi_{2}^{2}-b^{2}\right)\left(\xi_{3}^{2}-b^{2}\right) / b^{2}\left(b^{2}-a^{2}\right)\right]^{1 / 2} \\
w & =\xi_{1} \xi_{2} \xi_{3} / a b
\end{aligned}
$$

with $\xi_{1}>a>\xi_{2}>b>\xi_{3}>0$. We find that in these coordinates (3) becomes

$$
-\frac{1}{2} \sum_{i} \frac{\xi_{i}}{h_{i}^{2}} \frac{\partial \rho}{\partial \xi_{i}}=\frac{D}{h_{1} h_{2} h_{3}} \sum_{i} \frac{\partial}{\partial \xi_{i}}\left(\frac{h_{1} h_{2} h_{3}}{h_{i}^{2}} \frac{\partial \rho}{\partial \xi_{i}}\right)
$$

where

$$
h_{i}=\left[\left(\xi_{i}^{2}-\xi_{i+1}^{2}\right)\left(\xi_{i}^{2}-\xi_{i+2}^{2}\right) /\left(\xi_{i}^{2}-a^{2}\right)\left(\xi_{i}^{2}-b^{2}\right)\right]^{1 / 2},
$$

the variables $\xi_{1}, \xi_{2}, \xi_{3}$ being placed in cyclic order.

We shall be concerned with those solutions of (5) which are functions of one of the $\xi$ 's only, $\xi_{i}$. Because $h_{1} h_{2} h_{3} / h_{i}^{2}$ equals

$$
f\left(\xi_{i}\right)=\left[\left(\xi_{i}^{2}-a^{2}\right)\left(\xi_{i}^{2}-b^{2}\right)\right]^{1 / 2}
$$

times a function of $\xi_{i+1}$ and $\xi_{i+2}$, under these restrictions (5) becomes

$$
-\frac{1}{2} \xi_{i} f\left(\xi_{i}\right) \frac{d \rho}{d \xi_{i}}=D \frac{d}{d \xi_{i}}\left[f\left(\xi_{i}\right) \frac{d \rho}{d \xi_{i}}\right]
$$

The general solution of (8) is

$$
\rho\left(\xi_{i}\right)=c \int_{d}^{\xi_{i}} \frac{\exp \left[-t^{2} / 4 D\right]}{f(t)} d t
$$

where $c$ and $d$ are arbitrary constants.

The surface $\xi_{1}=\xi_{0}>a \geq b \geq 0$ corresponds to the growing ellipsoid in the original $x, y, z$ coordinate system with principal axes of length $2 t^{1 / 2}\left(\xi_{0}^{2}-a^{2}\right)^{1 / 2}, 2 t^{1 / 2}\left(\xi_{0}^{2}-b^{2}\right)^{1 / 2}$, $2 t^{1 / 2} \xi_{0}$ along the $x, y, z$ axes respectively. Hence the solution of (1) which equals $\rho_{0}$ on this surface and $\left(\rho_{0}+\rho_{s}\right)$ at infinity is from (9)

$$
\rho\left(\xi_{1}\right)=\rho_{s}+\rho_{0}\left\{1-\left[F\left(\xi_{1}\right) / F\left(\xi_{0}\right)\right]\right\},
$$

where

$$
F\left(\xi_{1}\right)=\int_{\xi_{1}}^{\infty} \frac{\exp \left[-t^{2} / 4 D\right] d t}{f(t)}
$$

with $f(t)$ defined in (7). At $t=0$ this corresponds to the uniform density $\rho=\rho_{0}+\rho_{s}$. The rate at which the ellipsoid grows is determined by $\xi_{0}$, its shape by the factors $\left[1-\left(a / \xi_{0}\right)^{2}\right]^{1 / 2},\left[1-\left(b / \xi_{0}\right)^{2}\right]^{1 / 2}$. To determine $\xi_{0}$ we shall require that the instantaneous velocity $\nabla$ in the $x, y, z$ coordinate system of a point $\left(u_{0}, v_{0}, w_{0}\right)$ on the surface $\xi_{i}=\xi_{0}$ shall have a component along the normal $\xi_{i}$ to the surface which is proportional to the normal component of the gradient of $\rho$ :

$$
\left(\rho_{c}-\rho_{s}\right)\left(\nabla \cdot \xi_{i}\right)=D\left(\xi_{i} \cdot \nabla \rho\right) .
$$

But from (2) we find

$$
\mathbf{r}=(x, y, z)=t^{1 / 2} \mathbf{s}
$$




$$
\begin{gathered}
\boldsymbol{\nabla}=\frac{1}{2} t^{-1 / 2} \mathbf{s}, \\
\nabla \rho=t^{-1 / 2} \nabla_{s} \rho .
\end{gathered}
$$

Moreover, if $\xi_{i}$ is a unit vector we can show from (4) that

$$
\left(\mathbf{s} \cdot \xi_{i}\right)=\xi_{i} / h_{i} \text {. }
$$

Hence (12) becomes

$$
\frac{1}{2}\left(\rho_{c}-\rho_{s}\right) \xi_{i}=D\left(\partial \rho / \partial \xi_{i}\right)
$$

to be satisfied for $\xi_{i}=\xi_{1}=\xi_{0}$. From (10), therefore, $\xi_{0}$ must satisfy

$$
\frac{1}{2}\left(\rho_{c}-\rho_{s}\right) \xi_{0} F\left(\xi_{0}\right)=-D \rho_{0} F^{\prime}\left(\xi_{0}\right),
$$

where $F^{\prime}(\xi)=(d / d \xi) F(\xi)$. This equation has a solution if $\rho_{0}<\left(\rho_{c}-\rho_{c}\right)$.

Physically (12) corresponds to the requirement that the flow of diffusing material to the surface be equal to the rate at which the amount of the new phase must be added locally if the surface is to advance with a velocity $\nabla$. In (12) $\rho_{c}$ represents the density of the diffusing material in the precipitate phase. In exhibiting (10) and (18), therefore, we have demonstrated the existence of a solution to (1) which satisfies this condition, with $\mathbf{v}$ given by (14), at every point of an ellipsoidal surface. The shape of the diffusion field for a density that is uniform at $t=0$ and constant at the surface during growth is therefore consistent with the growth of an ellipsoid of arbitrary but constant shape and dimensions proportional to $t^{1 / 2}$. $^{*}$

Another interesting solution is obtained from (9) and (17) if we use $\xi_{i}=\xi_{3}$. Surfaces of constant $\xi_{3}\left(0 \leq \xi_{3}<b \leq a\right)$ are hyperboloids of two sheets, with $\xi_{3}=0$ being the $u, v$ plane. Therefore

$$
\rho\left(\xi_{3}\right)=\rho_{0}+\left(\rho_{s}-\rho_{0}\right)\left[G\left(\xi_{3}\right) / G\left(\xi_{0}\right)\right]
$$

with

$$
G\left(\xi_{3}\right)=\int_{0}^{\xi_{3}} \frac{\exp \left[-t^{2} / 4 D\right] d t}{f(t)}
$$

is a solution of (1) which equals $\rho_{0}$ on the $x, y$ plane and $\rho_{s}$ on the hyperboloid $\xi_{3}=\xi_{0}<b \leq a$, or

$$
\frac{x^{2}}{a^{2}-\xi_{0}^{2}}+\frac{y^{2}}{b^{2}-\xi_{0}^{2}}-\frac{z^{2}}{\bar{\xi}_{0}^{2}}=-t .
$$

This hyperboloid has a trace on the $x, z$ plane which is a hyperbola with foci at $y=0$, $z= \pm a t^{1 / 2}$ and as asymptotes the lines $y=0, x= \pm\left[\left(a^{2}-\xi_{0}^{2}\right) / \xi_{0}^{2}\right]^{1 / 2} z$. On the $y, z$

*This analysis is not appropriate for the description of the growth of finite plates or rods of zero thickness (the limiting ellipsoidal forms), for the velocity of growth of the finite dimensions of the particle is found from (18) to be infinite in this limit. However, our ellipsoidal solutions reduce in this limit to those for planes or cylinders given in Sec. III. Thus the length or breadth of the particle becomes infinite in any finite time, and the thickness grows at a finite rate given by the simpler solutions for one or two dimensions. Such infinite growth velocities obviously make the solutions inadequate representations of any physical situation involving a finite three-dimensional particle, but this is not surprising since we do not expect the boundary conditions used in our analysis to be reasonable when any dimension of the particle is smaller than a few atomic diameters. 
plane the foci are at $x=0, z= \pm b t^{1 / 2}$ and the asymptotes $x=0, y= \pm\left[\left(b^{2}-\xi_{0}^{2}\right) /\right.$ $\left.\xi_{0}^{2}\right]^{1 / 2} z$.

If we impose the boundary condition (12) or (17) on the surface $\xi_{3}=\xi_{0}$, we find that $\xi_{0}$ must satisfy the equation

$$
\frac{1}{2}\left(\rho_{c}-\rho_{s}\right) \xi_{0} G\left(\xi_{0}\right)=D\left(\rho_{s}-\rho_{0}\right) G^{\prime}\left(\xi_{0}\right),
$$

where $G^{\prime}=(d G / d \xi)$. Therefore (19) and (22) correspond to the diffusion-limited transfer of material between one branch of a dissolving hyperboloidal surface of two sheets and the plane containing the conjugate axes of the hyperboloid. Thus if the density of diffusing material on this plane is maintained at a constant value $\rho_{0}$ by an external agency, the hyperboloid retains its shape as it dissolves, preserving its asymptotes and eccentricity while the distance between its foci and the plane increases in proportion to the square root of the time.

A second type of solution of (1) involving hyperboloidal surfaces corresponds to precipitation on such a surface instead of its dissolution as above. In this case we replace $t^{-1 / 2}$ in (2) by $(\tau-t)^{-1 / 2}$. The sign of the left-hand side of (3), (5), and (8) is correspondingly changed, as is that of the exponent in (9). The integrals corresponding to (10) and (11) diverge because of the positive sign of the exponential, so that we can not use a solution of this type to describe the dissolution of an ellipsoidal particle, at least not with the boundary condition of a given density of diffusing material at infinity. However, there is no such difficulty with (19) and (20), so that with a positive sign in the exponential this solution corresponds to transfer of material between the $x, y$ plane and the hyperboloid given by (21) with $t$ replaced by $(\tau-t)$. The shape of the hyperboloid is preserved, but at time $t=\tau$ the surface has become a cone with its apex touching the $x, y$ plane. The solution is valid only for $t<\tau$.

III. Limiting forms of the general solutions. Morse and Feshbach [6] have listed prescriptions whereby one can pass from ellipsoidal coordinates to most of the more familiar coordinate systems by appropriate stretching, compressing, and translating. If these or similar prescriptions are applied to the solutions (10) and (19) and the boundary conditions (18) and (22) as well as the coordinates, solutions to (1) appropriate to these simpler geometries are obtained. These agree for radially symmetric particles with the results of Rieck [1], Frank [2], and Zener [3].

As an example, if we set $\xi_{1}^{2}=a^{2}+{u^{\prime 2}}^{2} \xi_{2}^{2}=b^{2}+v^{\prime 2}$, and $\xi_{3}=w^{\prime}$ in (4), place $b=$ $a \sin \varphi$, and then let $a$ become infinite, we obtain $u=u^{\prime}, v=v^{\prime}, w=w^{\prime}$, or rectangular coordinates. Making these substitutions in (10), setting $\xi_{0}^{2}=a^{2}+u_{0}^{\prime 2}$, and taking the limit, we obtain an expression of the same form but with $F\left(\xi_{1}\right)$ replaced by

$$
F\left(u_{0}^{\prime}\right)=\int_{u_{0}}^{\infty} \exp \left[-u^{2} / 4 D\right] d u .
$$

This replacement in (18) also gives us the correct boundary condition. This is the usual solution [5] in one-dimensional diffusion for precipitation from a semi-infinite medium onto the plane $x=u_{0}^{\prime} t^{1 / 2}$.

Similar manipulations of (19) and (22) yield a one-dimensional result for the diffusion of matter between the plane $z=w_{0}^{\prime} t^{1 / 2}$ and the plane $z=0$. Finally, if we start from the second type of solution for hyperboloidal surfaces discussed above, we get a similar result for the planes $z=w_{0}^{\prime}(\tau-t)^{1 / 2}$ and $z=0$, all exponentials in the expressions corresponding to (19) and (22) having positive arguments in this case. 
The results obtained for the various coordinate systems and surfaces as limiting forms of the general ellipsoid can all be put in the form of (10) and (18) if the function $f(t)$ appearing in $F(\xi)$ is defined appropriately. The proper definitions are listed in Table $\mathrm{I}$, along with values of $a, b$, and $c$ which when substituted into the equation

$$
\frac{x^{2}}{\xi_{1}^{2}-a^{2}}+\frac{y^{2}}{\xi_{1}^{2}-b^{2}}+\frac{z^{2}}{\xi_{1}^{2}-c^{2}}=t
$$

define the surface over which $\xi_{1}$ and $\rho\left(\xi_{1}\right)$ are constant. We restrict $\xi_{1}$ to values greater than $a, b$ and $c$ when these numbers are finite. An infinite value for $a, b$, or $c$ simply deletes the corresponding term from (24).

TABLE I.

Ellipsoidal solutions in special coordinate systems.

\begin{tabular}{|c|c|c|c|c|}
\hline \multirow[t]{2}{*}{ Coordinate System } & \multicolumn{3}{|c|}{ Surface Parameters* } & \multirow[t]{2}{*}{$f(t) \dagger$} \\
\hline & $a$ & $b$ & $c$ & \\
\hline Ellipsoidal & $\dot{a}$ & $b$ & 0 & {$\left[\left(t^{2}-a^{2}\right)\left(t^{2}-b^{2}\right)\right]^{1 / 2}$} \\
\hline Rectangular & 0 & $\infty$ & $\infty$ & 1 \\
\hline Oblate spheroidal & $a$ & 0 & 0 & $t\left[t^{2}-a^{2}\right]^{1 / 2}$ \\
\hline Prolate spheroidal & $a$ & $a$ & $\mathbf{0}$ & {$\left[t^{2}-a^{2}\right]$} \\
\hline Elliptic cylinder & $a$ & 0 & $\infty$ & {$\left[t^{2}-a^{2}\right]^{1 / 2}$} \\
\hline Circular cylinder & 0 & 0 & $\infty$ & $t$ \\
\hline Spherical & 0 & 0 & $\mathbf{0}$ & $t^{2}$ \\
\hline
\end{tabular}

*See Eq. (24) of text.

†See Eqs. (10), (11), and (18) of text.

TABLE II.

Hyperboloidal solutions in special coordinate systems.

\begin{tabular}{|c|c|c|c|}
\hline \multirow[t]{2}{*}{ Coordinate System } & \multicolumn{2}{|c|}{ Surface Parameters* } & \multirow[t]{2}{*}{$f(t) \dagger$} \\
\hline & $a$ & $b$ & \\
\hline Ellipsoidal & $a$ & $b$ & {$\left[\left(a^{2}-t^{2}\right)\left(b^{2}-t^{2}\right)\right]^{1 / 2}$} \\
\hline Rectangular & $\infty$ & $\infty$ & 1 \\
\hline Prolate spheroidal & $a$ & $a$ & {$\left[a^{2}-t^{2}\right]$} \\
\hline Elliptic cylinder & $\infty$ & $b$ & {$\left[b^{2}-t^{2}\right]^{1 / 2}$} \\
\hline
\end{tabular}

*See Eq. (21) of text.

†See Eq. (19), (20), and (22) of text.

The hyperboloidal solutions (19) have interesting and distinct limiting forms only for the rectangular, prolate spheroidal, and elliptic cylinder coordinates. These solutions and the boundary condition all have the form of (19) and (22) with $f(t)$ in (20) appropriately defined. The proper definitions are listed in Table II together with values of $a$ and $b$ which when substituted into (21) (with $\xi_{3}$ replacing $\xi_{0}$ ) define the surface over which $\xi_{3}$ and $\rho\left(\xi_{3}\right)$ are constant. We restrict $\xi_{3}$ to values between zero and the lesser of $a$ and $b$.

If the argument of the exponential in (20) is made positive and $t$ replaced by $(\tau-t)$ in (21), then (19), (21), (22) and Table II give the hyperboloidal solutions for which the scale of the geometry is shrinking instead of expanding. 
Other types of coordinate systems may be obtained from the ellipsoidal coordinates, but either the limiting surface of interest is identical with one we have already listed (for conical coordinates, for example, the interesting surfaces are spheres) or the limit of (10) or (19) is indeterminate (for paraboloidal and similar systems). In the latter case we can show directly that for these systems (5) does not separate in the sense that if $\rho$ is a function of only one coordinate the other coordinates disappear from the equation.

Evaluation of the integrals appearing in our solutions can not be done simply, although those for rectangular and spherical coordinates can be expressed in terms of tabulated error functions. Frank [2] has given tables for the evaluation of (11) for spherical and circular cylinder coordinates. Since $t^{2}>a^{2}>b^{2}$ in integrals of type (11), the more complicated integrals can be evaluated by expanding the factor multiplying the exponential in a power series in $(1 / t)$ and tabulating integrals of the form $\int_{x}^{\infty} t^{-n}$ $\exp \left(-t^{2}\right) d t$. In integrals of type (20), $t^{2}<b^{2}<a^{2}$, so that use of a power series in $t$ is appropriate. Also, if $\xi / D^{\frac{1}{2}}$ is sufficiently small, the exponential factor in the integrand can be replaced by unity. The resulting integrals are then integrable in terms of simple functions, the ellipsoidal result becoming an elliptic integral. This approximate procedure is not satisfactory for cylindrical and rectangular coordinates, since (11) diverges in these cases if the exponential is omitted.

In numerical evaluations of these solutions it is convenient to note that the shape of the surface on which the boundary condition is satisfied is fixed by the parameters $a^{2} / \xi_{0}^{2}$ and $b^{2} / \xi_{0}^{2}$. With these chosen, the boundary condition (18) or (22) is then an equation for $\xi_{0}$, which determines the rate of growth of the surface.

IV. Generalizations. I't is possible to generalize our solutions to considerably more complicated situations analogous to those for a plane interface discussed by Danckwerts [4] and Crank [5]. Thus if the diffusion constant has a discontinuity at a concentration $\rho_{c}$, we can use one expression of the form (9) in the region $\rho<\rho_{c}$, another for $\rho>\rho_{c}$. Conditions of continuity, the shape of the precipitate surface, a growth condition like (17) at the surface, and prescribed values of $\rho$ at infinity, the discontinuity, and the surface then may be used to determine the parameters and integration constants in the solutions in the two regions. Alternatively, we can specify the normal velocity of two confocal surfaces in terms of the rate at which the diffusing material is transported across each surface, in analogy with Danckwerts' "Class A" problems [4, 5]. If we do not relate the surface velocity to the rate of transport but instead determine our solutions by specifying densities at the surfaces, we have the generalization of "Class B" problems. Finally, we can treat the problem in which the growth of the new phase depends on the diffusion of two or more substances. This was solved by Frank for radial symmetry [2].

V. Anisotropic diffusivity. We shall now show that the preceding solutions are also appropriate when the diffusivity is anisotropic. Choosing coordinate axes such that the diffusivity tensor is in diagonal form, we have in place of (1).

$$
\frac{\partial \rho}{\partial t}=D_{1} \frac{\partial^{2} \rho}{\partial x^{2}}+D_{2} \frac{\partial^{2} \rho}{\partial y^{2}}+D_{3} \frac{\partial^{2} \rho}{\partial z^{2}} .
$$

The transformation

$$
\begin{aligned}
& x^{\prime}=x / D_{1}^{1 / 2}, \\
& y^{\prime}=y / D_{2}^{1 / 2}, \\
& z^{\prime}=z / D_{3}^{1 / 2},
\end{aligned}
$$


then brings (25) to the form of (1) in terms of the primed coordinates, with $D=1$.

An ellipsoidal or hyperboloidal surface centered at the origin is described by the equation

$$
A x^{2}+B y^{2}+C z^{2}+D x y+E y z+F z x-G=0,
$$

with suitable values of the coefficients. The principal axes of this surface are in general different from those of the diffusivity tensor. The boundary condition for the diffusionlimited growth of this surface is now

$$
\left(\rho_{c}-\rho_{s}\right)(\mathbf{v} \cdot \mathbf{n})=\mathbf{n} \cdot \mathfrak{D} \nabla \boldsymbol{\rho}
$$

in place of (12), $\nabla$ being as usual the velocity of a point on the surface, $\mathfrak{D}$ the diffusivity tensor

$$
\mathfrak{D}=\left(\begin{array}{ccc}
D_{1} & 0 & 0 \\
0 & D_{2} & 0 \\
0 & 0 & D_{3}
\end{array}\right)
$$

and $\mathbf{n}$ the vector

$$
\mathbf{n}=(2 A x+D y+F z, 2 B y+D x+E z, 2 C z+E y+F x)
$$

which is normal to (27) at the point $\mathbf{r}=(x, y, z)$. The length of $\mathbf{n}$ is of no concern since (28) is linear in the components of $\mathbf{n}$.

If the surface preserves its shape during growth, $\mathbf{\nabla}$ is proportional to the position vector $\mathrm{r}$ :

$$
\mathbf{v}=K f(t) \mathbf{r} \text {. }
$$

Substituting (29), (30), and (31) into (28) and introducing the change of variable (26), we obtain the boundary condition appropriate to shape-conserving growth expressed in the primed coordinates,

$$
\left(\rho_{c}-\rho_{s}\right)\left(\nabla^{\prime} \cdot \mathbf{n}^{\prime}\right)=\left(\mathbf{n}^{\prime} \cdot \nabla^{\prime} \rho\right)
$$

Here $n^{\prime}$ is the vector

$$
\begin{aligned}
\mathbf{n}^{\prime}= & {\left[2 A D_{1} x^{\prime}+D\left(D_{1} D_{2}\right)^{1 / 2} y^{\prime}+F\left(D_{1} D_{3}\right)^{1 / 2} z^{\prime},\right.} \\
& 2 B D_{2} y^{\prime}+D\left(D_{1} D_{2}\right)^{1 / 2} x^{\prime}+E\left(D_{2} D_{3}\right)^{1 / 2} z^{\prime}, \\
& \left.2 C D_{3} z^{\prime}+E\left(D_{2} D_{3}\right)^{1 / 2} y^{\prime}+F\left(D_{1} D_{3}\right)^{1 / 2} x^{\prime}\right],
\end{aligned}
$$

which is normal to the surface into which (27) is transformed by (26) in the primed coordinates. Also

$$
\mathbf{\nabla}^{\prime}=K f(t) \mathbf{r}^{\prime} .
$$

This boundary condition (32) is identical with (12) (with $D=1$ ), however, if $\nabla^{\prime}$ is identified as the velocity of a point on the surface in the primed coordinates. ${ }^{*}$ Since in this coordinate system the diffusivity is isotropic, the solution of (1) and (32) for constant initial density is given immediately by our earlier results. These determine $K$ and $f(t)$

*Note that $\nabla^{\prime}$ is not the vector obtained by substituting from (26) into the components of $v$ in (31). 
$\left(=t^{-1}\right)$ in (34), and these in turn determine $\nabla$ in (31). Thus on transforming back to the original coordinates we have the solution to the problem of the growing surface with anisotropic diffusion, and we see that it indeed corresponds to the preservation of the shape of the surface during growth.* Surfaces of constant concentration are not confocal with this boundary surface in the original coordinates, although they are in the coordinates for which the diffusivity is isotropic.

VI. Discussion. Of the solutions of the time-dependent diffusion equation which we have exhibited, those pertaining to the growth of ellipsoidal particles are of particular interest in the theory of diffusion-limited precipitation from supersaturated solution. As the author has pointed out elsewhere in a discussion of various aspects of precipitation theory [7], the existence under appropriate conditions of solutions corresponding to the growth of ellipsoidal particles of constant shape indicates that in these situations the diffusion field is not responsible for any change that may actually occur in the particles' shape. A change should be attributed to some other cause, therefore. This result contrasts with views that have been widely held in the absence of accurate solutions of the diffusion equation for asymmetric particles or an anisotropic diffusivity. Of course, one can not use the diffusion equation or simple boundary conditions to describe the initial stages of formation of an actual precipitate particle, and our solutions are exact for a uniform initial solute distribution only under the physically artificial restriction that the particles have zero dimensions at $t=0$. Our solutions are, however, useful in approximating real diffusion-limited growth when the particles have reached a size that permits the neglect of atomic fluctuations and other complications. of the nucleation process and when transient effects associated with finite initial dimensions of the particles have substantially vanished.

The usefulness of these solutions for asymmetric surfaces is broadened by their applicability to problems for which the diffusivity is anisotropic. Thus the growth of a radially symmetric particle with anisotropic diffusion is mathematically equivalent to growth of an asymmetric particle with isotropic diffusion. Indeed, the general problem of an arbitrarily oriented ellipsoidal particle of zero initial dimensions with anisotropic diffusion can be solved exactly by means of this transformation, as we have shown. In all cases we find that the surface preserves its shape as it grows, the rate of transport of material across each point of the surface sufficing to give a local surface growth velocity appropriate to this result.

The solutions for hyperboloidal surfaces have no immediate practical application

\footnotetext{
*This result may appear at first sight to be incorrect in the limiting case in which one or more components of the diffusivity tensor are identically zero. Thus if one interprets the vanishing of $D_{1}$ and $D_{2}$ in (29) as requiring that no transport occur except in the $z$-direction, one must conclude that a growing particle extends its dimensions only parallel to the $z$-axis, so that its shape is not preserved. However, a discontinuity in the solute density (or equivalently an infinite concentration gradient) then exists on the cylinder which is parallel to the $z$-axis and tangent to the particle. On the other hand, the solution of the text predicts in the limit $\left(D_{1}, D_{2} \rightarrow 0\right)$ that, for example, a sphere of zero initial radius grows at a finite rate and remains spherical. Surfaces of constant concentration are spheroids of revolution which are tangent to the sphere and have their major axes along the $z$-direction. An infinite concentration gradient therefore exists at the circle of tangency, but in this limiting case the product of a vanishing component of diffusivity and an infinite gradient leads to a finite current density. The different predictions for the limiting case therefore depend upon how one deals with an infinite concentration gradient. The result of the text is valid if we associate with an infinite gradient a current density obtained by approaching the limit from finite diffusivities.
} 
so far as the author is aware. They do correspond to the diffusion-limited dissolution of a conical or wedge-shaped surface placed initially with its apex touching a plane on which the density is maintained constant by an external agency. However, our solutions require that the density vary appropriately in the region between the plane and the cone even at $t=0$, and this condition would be difficult to achieve in practice.

\section{REFERENCES}

1. R. Rieck (1924), quoted by Frank in Ref. 2 below

2. F. C. Frank, Proc. Roy. Soc. (London) A201, 586 (1950)

3. C. Zener, J. Appl. Phys. 20, 950 (1949)

4. P. V. Danckwerts, Trans. Faraday Soc. 46, 701 (1950)

5. J. Crank, The Mathematics of Diffusion, Clarendon Press, Oxford, Chap. VII, 1956

6. P. M. Morse and H. Feshbach, Methods of Theoretical Physics, vol. I, McGraw-Hill, New York, 1953, pp. 511-515

7. F. S. Ham, J. Phys. Chem. Solids, 6, 335 (1958) 